\title{
Micro-mechanical Resonators for Dynamically Reconfigurable Reduced Voltage Logic Gates
}

\author{
K. N. Chappanda ${ }^{1}$, S. Ilyas ${ }^{1}$, and M. I. Younis ${ }^{1^{*}}$ \\ ${ }^{1}$ Nano/Micro Mechanics and Motion Laboratory, Physical Science and Engineering Division, King \\ Abdullah University of Science and Technology, Thuwal, 23955-6900, Kingdom of Saudi Arabia. \\ *Corresponding author e-mail: mohammad.younis@kaust.edu.sa
}

\begin{abstract}
Due to the limitations of transistor based logic devices such as their poor performance at elevated temperature, alternative computing methods are being actively investigated. In this work, we present electromechanical logic gates using electrostatically coupled in-plane micro-cantilever resonators operated at modest vacuum conditions of 5 Torr. Operating in the first resonant mode, we demonstrate 2-bit XOR, 2and 3-bit AND, 2- and 3-bit NOR, and 1-bit NOT gates; all condensed in the same device. Through the designed electrostatic coupling, the required voltage for the logic gates is reduced by $80 \%$, along with the reduction in the number of electrical interconnects and devices per logic operation (contrary to transistors). The device is dynamically reconfigurable between any logic gates in real time without the need for any change in the electrical interconnects and the drive circuit. By operating in the first two resonant vibration modes, we demonstrate mechanical logic gates consisting of two 2-bit AND and two 2-bit XOR gates. The device is tested at elevated temperatures and is shown to be functional as a logic gate up to $150{ }^{\circ} \mathrm{C}$. Also, the device has high reliability with demonstrated lifetime greater than $5 \times 10^{12}$ oscillations.
\end{abstract}

Keywords: Coupled resonators, Electromechanical logic gates, Reconfigurable logic gates, High temperature. 


\section{Introduction}

There is an increasing interest in alternative logic gates due to the limitations of complementary-metaloxide-semiconductor (CMOS) transistors-based logic gates, such as their inability to dynamically reconfigure their functionality in real time, inability to operate at high temperatures/ionizing environments, and their high off-state power consumption. One approach to overcome these limitations is through mechanical logic gates. With the advances in device fabrication technologies reaching the sub-nanometer regime [1], the interest in scalable mechanical logic gates based on micro/nano electromechanical systems (M/NEMS) devices has been accelerating recently [2-19]. Although current M/NEMS devices lag behind transistors in terms of speed, size, power and device lifetime, they promise several advantages. A complete logic function can be realized in a single M/NEMS device, which otherwise would require multiple interconnected transistors. M/NEMS logic devices use electro-static [2-8] and electro-dynamic [9-19] actuation techniques. High temperature and multiple logic gate functionality have been demonstrated in both static $[2,6]$ and dynamic $[14,15]$ based mechanical logic gates, which are limited in transistors. Further, dynamic logic devices have several advantages over static devices. Multiple logic gate functionalities can be achieved using simple device structures, such as cantilevers [14], doubly-clamped beams [12, 15], and membranes [16]. Also, dynamic devices eliminate the need for different electrical interconnects to realize multiple logics within a single structure [12-15]. Another key advantage is that dynamic devices operate contact free while switching between 'ON' and 'OFF' Boolean states, and hence have longer lifetime compared to static devices that rely on physical contacts between electrodes during switching [20]. Also, due to the need to trigger pull-in in static actuation, higher actuation voltages are required in comparison to dynamic actuation.

In transistors and static actuation based M/NEMS logic gates, the 'presence (absence)' of DC current is used to define the ' $1(0)$ ' Boolean output states. Alternately, dynamic actuation based M/NEMS logic gates use the 'presence (absence)' of motional current due to the 'resonating (non-resonating)' states of the device for defining the ' $1(0)$ ' Boolean output states [9-19]. Masmanidis et al. [17] demonstrated the first scalable dynamically actuated mechanical logic gate (XOR) using two electrically isolated but 
mechanically connected (in an L-shape) piezoelectric nano-cantilevers. The presence/absence of the AC inputs at the electrodes of the cantilevers were used to define the ' $1 / 0$ ' Boolean gate inputs, respectively. AND/NAND and OR/NOR logic gates were demonstrated using doubly clamped nano-beam operating in the nonlinear regime [12]. Switching between the two output states (operating in the non-linear hysteresis regime) is modulated by gate input DC input voltage and mediated by the white noise floor. The device is switched between AND/NAND and OR/NOR logic gates by modulating the amplitude of input AC drive signal. Mahboob et al. [13] demonstrated a universal logic gate with a piezoelectric GaAs/AlGaAs based doubly clamped micro-beams. AND, OR, XOR, and multi-bit logic gate functions were achieved using a parametrically excited resonator. AC input signals of different frequencies were used to define the Boolean gate inputs, where the presence/absence of the right mixed frequency signal results in a resonating or nonresonating output state. Similarly, XOR and OR logic gates were demonstrated using different vibration modes of piezoelectric GaAs/AlGaAs based membrane structure [16]. Using the bi-stable regime of a comb-drive MEMS resonator, the device was demonstrated to function as a memory (without feedback control loop) as well as an OR logic gate (with feedback control loop) [9]. Using electro-thermally modulated frequency of doubly clamped micro-beams, NOR, NOT, XNOR, XOR, and AND logic gate were demonstrated, where different drive frequency was used for realizing different logic gates [15]. The resonance frequency of the device was tuned by changing the spring constant by electro-thermal heating, allowing the device to switch between the two output states at the drive frequency. Similarly, a single-bit binary comparator and a single-bit 4-to-2 encoder, was demonstrated using three and two electrically interconnected electro-thermally modulated micro-beams, respectively [18]. DC voltage induced electrothermal heating was used as gate inputs. Further, a Fredkin gate using four electrically interconnected nanoresonators have been demonstrated [11]. The switching between the output states was performed by electrostatic DC bias based tuning of resonance frequency. Also, using a nano-cantilever with two drive electrodes, XOR, AND, NOR, OR and NOT logic gates have been demonstrated, operating up to $100{ }^{\circ} \mathrm{C}$ [14]. Different logic gates were achieved by using different gate input voltages at a single operating 
frequency. Furthermore, the activation and deactivation of combination resonances arising due to frequency mixing have been used to perform AND, NAND, OR, NOR, and a tri-state logic gate [10].

\section{Device Principle}

In this work, we operate the device in the linear regime and rely on switching between the output states using the DC bias induced shift in resonance frequency. Although DC induced frequency shift has been studied extensively, their use for realizing logic gates have been limited. This works aims to maximize the influence of the DC bias and the frequency shift through the utilization of two electrostatically coupled resonating structures, of which, one of the movable beam itself is used as a drive electrode [21]. Through the electrostatic coupling, the effective spring constant of the system becomes more sensitive compared to the traditional configuration of fixed-movable parallel-plate actuators. This further enhances the voltage bias induced shift in the resonance frequency, and hence reduces the required gate input voltages for realizing logic devices. Fig. 1 shows a schematic illustrating this point, where the coupled system has higher shift in resonance frequency compared to the classical parallel-plate configuration. Operating in the first resonant mode, 2-bit XOR, 2- and 3-bit AND, 2- and 3-bit NOR, and 1-bit NOT logic gates with reduced gate input voltages, are demonstrated in a single device. Also, most previously reported mechanically [22], electrically [23] and electrostatically [21] coupled resonators use beams, which are identical to each other. However, it is not possible to have perfectly identical beams due to fabrication anomalies, and the presence of identical beams will cause the first two resonant modes to overlap with each other [21]. Here, the two resonating cantilevers are designed to be different in terms of their lengths (100 and $105 \mu \mathrm{m})$, which sufficiently split and separate the first two resonant modes [21, 24], and thus provide two resonance peaks (dual-band) for realizing logic gates. Utilizing the two resonant modes, two AND and two XOR logic gates are also demonstrated. Note that the second mode of the single cantilever (uncoupled) can also provide the second resonance band. However, the second mode frequency is more than 7 times higher than the first resonant mode and requires much higher actuation voltages to achieve detectable signal [25]. The device is dynamically reconfigurable between any logic gate function without the need for any change in the drive 


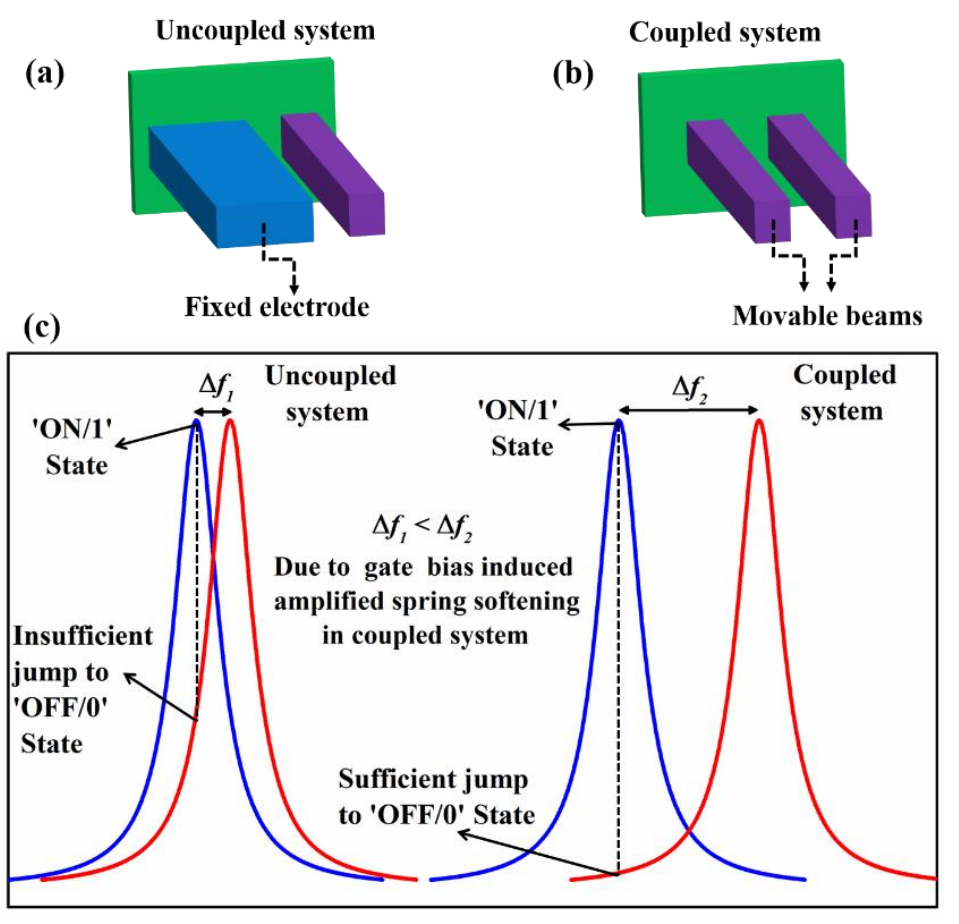

Fig. 1 Schematic illustrating the working principle of the device. (a) Uncoupled system with fixed-movable parallel-plate actuator. (b) Coupled system with movable-movable parallel-plate actuators. (c) Coupled system has higher shift in resonance frequency, which allows a decisive switch between the ' 1 ' and ' 0 ' output states.

circuit. Achieving such dynamically reconfigurable multi-logic functionality using transistors requires a large number of electrically interconnected devices, leading to higher energy losses and delayed computing. The electrical drive circuit used here is relatively simple, consisting of a two-port configuration. Most previously reported works use clamped-clamped structures, which are prone to temperature induced axial stresses that may cause significant shift in the resonance frequency. Cantilever beams are stress free structures that do not experience internally induced axial loads making them almost immune to temperatureinduced frequency shifts. Hence, they are suitable candidates for high temperature applications. Here, we experimentally demonstrate an XOR logic operation at $150^{\circ} \mathrm{C}$ as a case study.

\section{Device Fabrication and Experimental setup}

\subsection{Device fabrication}

Fig. 2(a) shows a false color scanning electron microscope (SEM) micrograph of the electrostatically 
coupled resonator. The device consists of two in-plane parallel cantilever beams that are slightly different in lengths (100 and $105 \mu \mathrm{m})$ along with two corresponding fixed electrodes (F1 and F2) on either side. The beams have a thickness of $3 \mu \mathrm{m}$, height of $25 \mu \mathrm{m}$, and a gap of $2 \mu \mathrm{m}$ separating them. The device is fabricated from highly doped $\mathrm{N}$-type Si-on-insulator (SOI) wafer with $25 \mu \mathrm{m}$ device layer and $2 \mu \mathrm{m}$ buried oxide layer. 50/250 nm Cr/Au layer was sputter deposited and patterned using optical lithography and liftoff, forming low resistance contacts pads for interfacing the fixed electrodes and the beams. The electrodes and beams were then defined using standard optical lithography followed by deep-reactive ion etching (DRIE) of Si. The cantilevers were released to form free standing resonating structures by etching the buried oxide under the beams using HF vapor etch at $40^{\circ} \mathrm{C}$. Fig. 2(b) shows a schematic of the device with its designed dimensions. Fig. S1 (see supplementary information) shows the schematic of the steps used for fabricating the device. The variation in lateral dimensions and layer thicknesses of the fabricated beams were $<150 \mathrm{~nm}$, from the desired/designed dimensions.

\subsection{Experimental setup}

The device was characterized in a ST-500 temperature controlled vacuum probe station from Janis Research (Fig. S2 \{see supplementary information\}). Fig. 3(a) shows the drive configuration of the coupled system consisting of two-port open loop drive circuit, used for characterizing the electromechanical resonator. The shorter/stiffer beam (B1) was biased with gate voltage $\mathrm{V}_{\mathrm{B}}$, while the fixed electrode (F1) and the longer/less stiffer beam (B2) were configured as the sense and drive electrodes, respectively. The alternating current (AC) frequency sweep $V_{A C}$ was provided using the network analyzer (E5071C ENA, from Keysight), for measuring $\mathrm{S}_{21}$ parameters. An AC voltage of load $V_{A C}=70 \mathrm{mV}_{\mathrm{RMS}}(-10 \mathrm{dBm})$ was used for all the frequency sweeps. The electrical output from the sense electrode is amplified using a SR560 (from Stanford Research) low noise amplifier (LNA), which amplifies the motional output current of the device to a voltage level that is detectable by the network analyzer. Fig. 3(b) shows the drive configuration for actuating B2 as an uncoupled system. Uncoupled system corresponds to actuating the beams independent of each other. This is realized by DC biasing the fixed drive electrode F2 through a bias tee. All the frequency sweep readings are acquired in the forward direction. Applying a gate bias generates an 
(a)

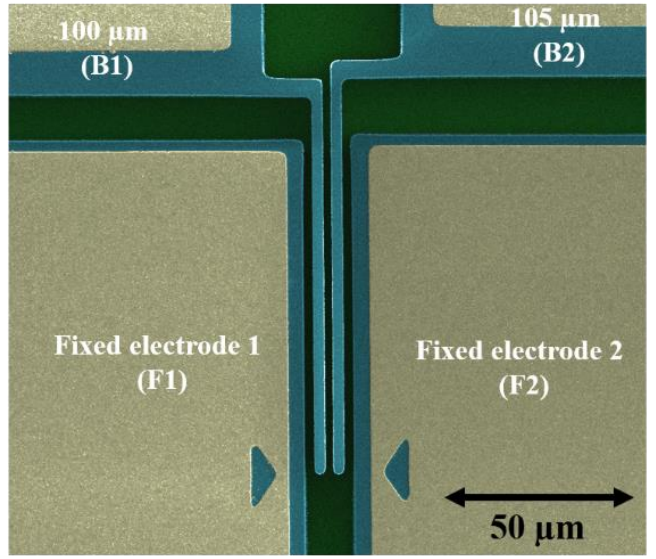

(b)

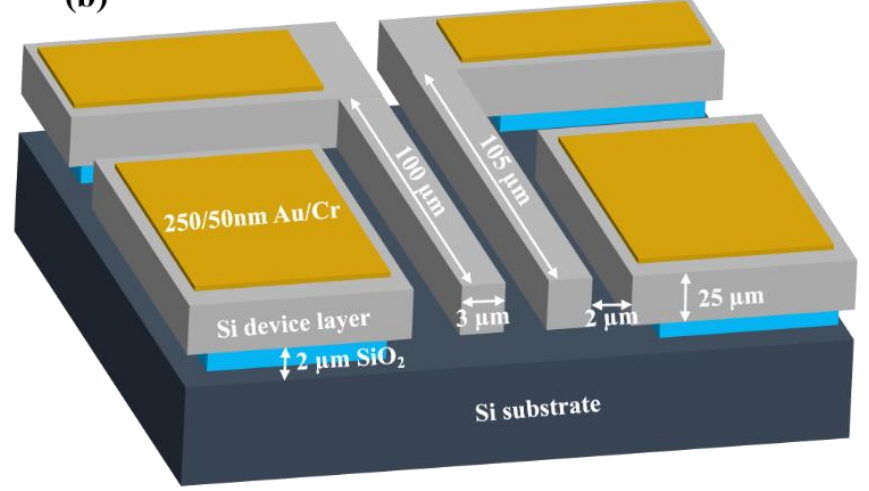

Fig. 2 Device structure and dimensions. (a) False colored SEM micrograph of electrostatically coupled two in-plane micro-cantilevers. The device was fabricated from Si-on-insulator (SOI) wafers using optical lithography and surface micromachining. The device consists of two near-identical cantilevers slightly different in lengths (100 and $105 \mu \mathrm{m})$. (b) A schematic showing the device with its dimensions.

electrostatic force between the two beams. The device was characterized at 5 Torr vacuum pressure and at room temperature unless otherwise specified. For higher temperature characterization the sample stage in the vacuum probe station was heated. A secondary inbuilt temperature sensor was used to ensure that the desired temperature was attained

\section{Results and discussions}

\subsection{Electrical characterization}

We electrically characterized the device for realizing the electro-mechanical logic gates. The device has two resonant modes. In the first resonant mode (M1), the two beams vibrate out-of-phase whereas in the second resonant mode (M2), the two beams vibrate in-phase [21] (Fig. 3(c)). Fig. 3(d) shows the electrically measured frequency response curves of the coupled resonator at $55 \mathrm{~V}$ gate bias voltages $V_{B}$ showing the two resonant modes. To understand the effect of electrostatic coupling, we compared the shift in resonance frequencies of the coupled and uncoupled systems. Fig. 4 shows the comparison of the shift in resonance frequency, when $V_{B}$ is varied from 20 to $60 \mathrm{~V}$. Mode 1 and the corresponding beam B2 experience a shift in resonance frequency by 56.9 , and $16.9 \mathrm{kHz}$, respectively. Mode 1 has a 335\% higher 
(a)

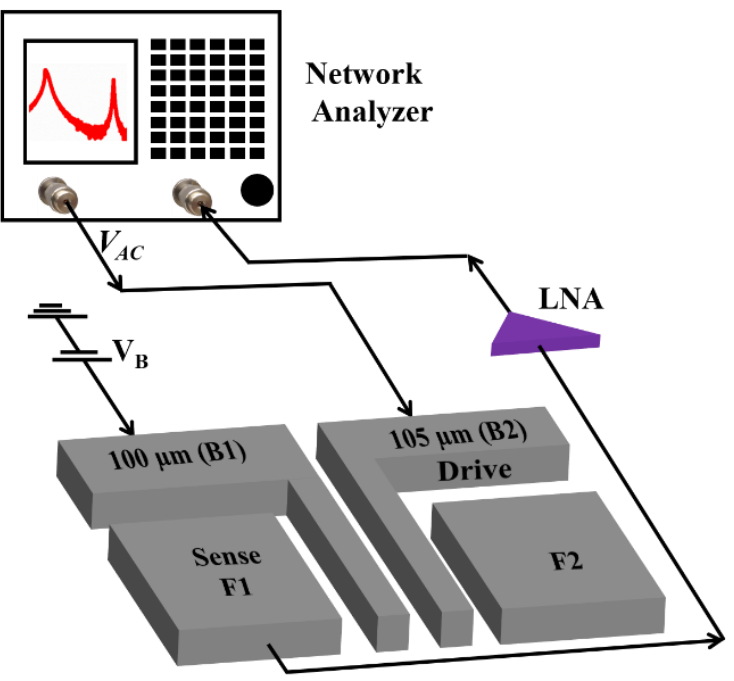

(c)

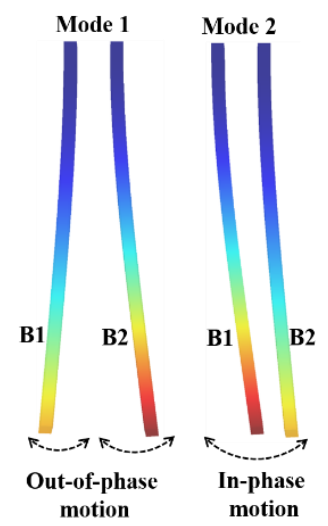

(b)
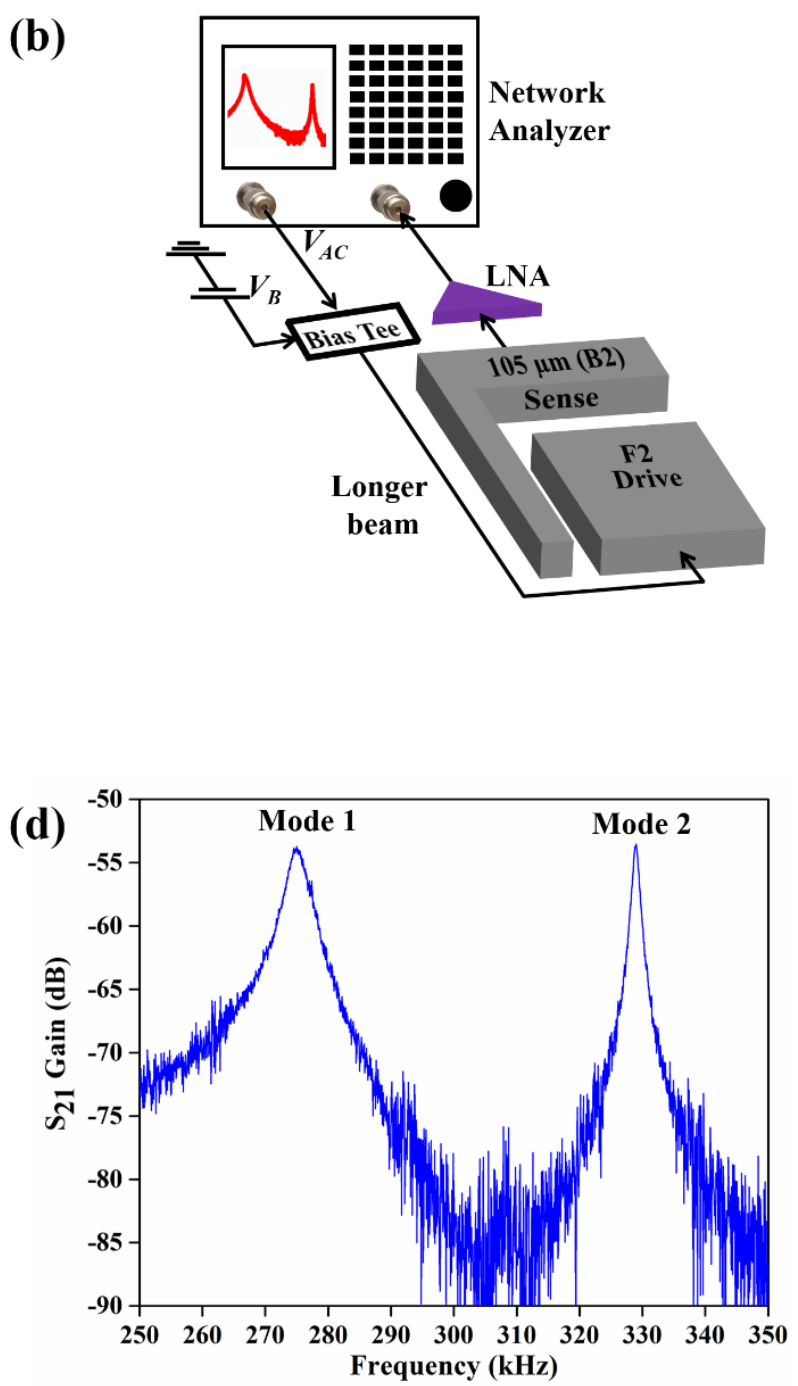

Fig. 3 Schematic of the two electrical drive circuits used for characterizing the mechanical resonators. (a) Coupled resonator setup, where the shorter beam (B1) is biased with the gate voltage, the longer beam (B2) acts as the drive electrode, and the fixed electrode (F1) as the sense electrode. (b) Uncoupled resonator setup, where B2 is actuated independent of B1. (c) The first two resonant modes shapes of the coupled beams, obtained from a finite element analysis, where in mode 1 the beams are out-of-phase and in mode 2 the beams are in-phase. (d) Frequency response of the coupled system at $V_{B}=55 \mathrm{~V}$.

shift in resonance frequency when compared to the resonance of corresponding uncoupled longer beam. This larger shift in resonance frequency is due to the increased effective electrostatic force, in which the coupled beams have $30.3 \%$ lower effective spring constant voltage when compared to the uncoupled longer beam [21]. This increases the operating frequency bandwidth for realising the logic gate functions in 


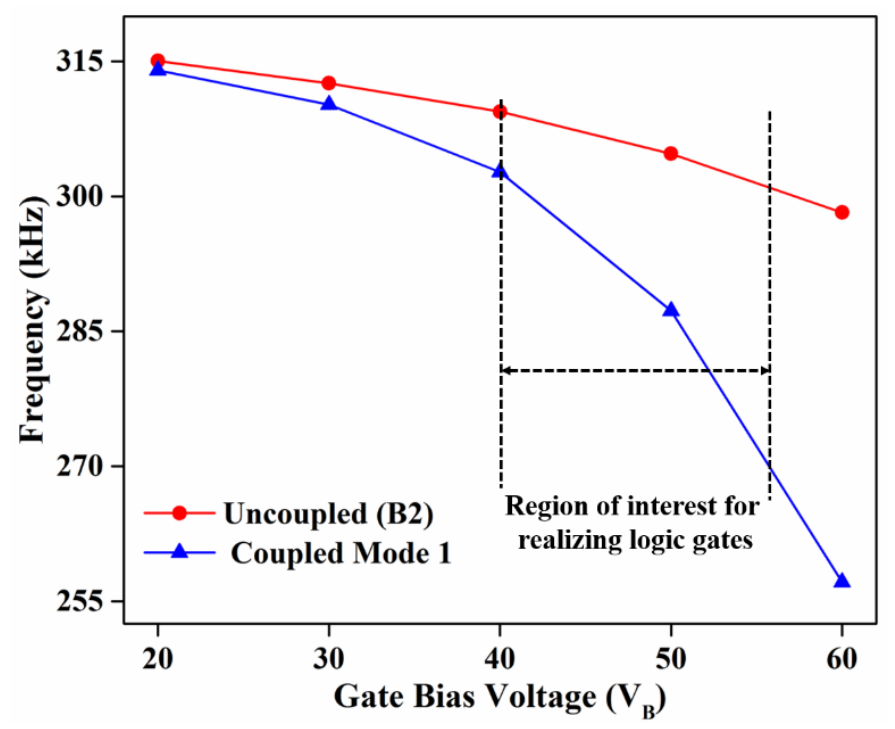

Fig. 4 Comparison of the shift in resonance frequency of the coupled and uncoupled systems from 20 to 60 V DC bias $V_{B}$.

comparison to the uncoupled system. The number of operating frequency points is defined by the width of resonant band. Note that the frequency bandwidth refers to the range of resonance frequencies up to which the device can be tuned by the gate bias induced spring softening.

\subsection{Reduced-voltage electro-mechanical logic gates}

One of the important criteria to enhance the performance of logic devices is to reduce energy cost per logic operation. This can be achieved by reducing the gate input voltages required per logic operations. The minimum gate voltages must be sufficient enough to completely shift the resonant bandwidth of the device (to switch between ' 0 ' and ' 1 ' states, Fig. 1). From Fig. 4 , it can be seen that at $V_{B} \sim 40 \mathrm{~V}$ and above, the coupled device has $400 \%$ higher shift in resonance frequency compared to B2. Using the voltage induced amplified spring softening in the coupled system at $40 \mathrm{~V}$ and above, we realise dynamically reconfigurable multi-functional mechanical logic gates with reduced input gate voltages, compared to achieving the same using a classical parallel-plate capacitor (which requires higher voltage). Fig. 5(a) shows the electrical drive circuit for realising 1-, 2- and 3-bit logic gates. A DC bias gate input with three voltage levels $\left(I_{1}, I_{2}, I_{3}\right)$ is interfaced to the drive electrode using a bias tee. With this configuration, the gate input voltage opposes the 
(a)

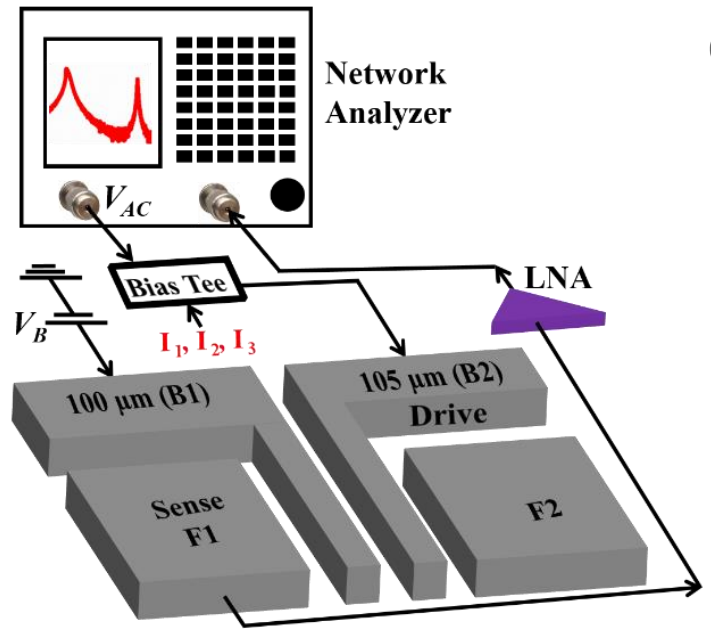

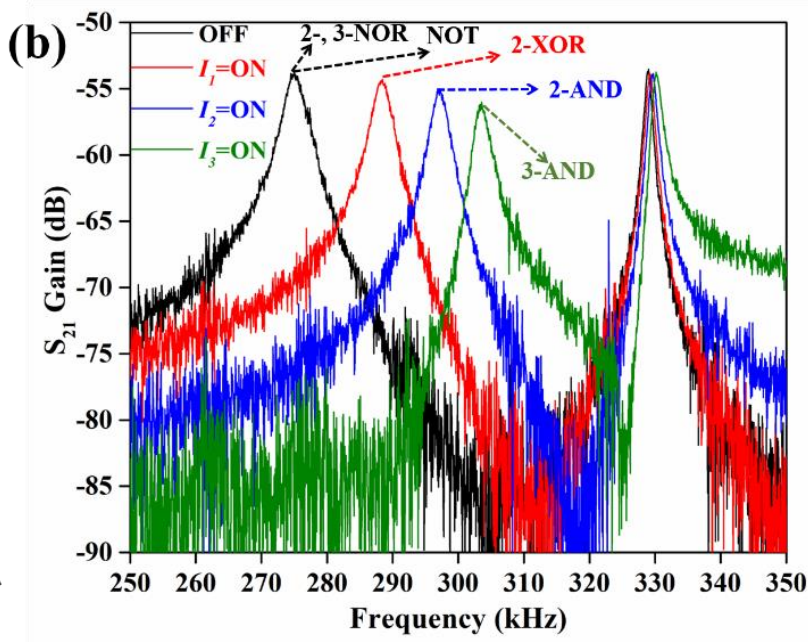

Fig. 5 (a) Schematic of the drive circuit for realizing the reduced gate voltage mechanical logic gates. A DC gate bias with three voltage levels $\left(I_{1}, I_{2}, I_{3}\right)$ are interfaced using a bias tee for realising 1-, 2- and 3-bit logic gates. (b) Frequency response of the coupled system at the three input voltage levels $\left(I_{1}, I_{2}, I_{3}\right)$.

bias voltage $V_{B}$, and induces shift in resonance curves. $V_{B}$ is fixed at $55 \mathrm{~V}$ for harvesting the higher shift in resonance frequency of mode 1 . Here, the required gate input voltage for performing the logic operations is $5 \mathrm{~V}$ per bit. When the gate input is set at $I_{l}(=5 \mathrm{~V})$, it represents ' 1 ' input for the 1-bit logic gate, ' 0,1 ' or ' 1,0 ' inputs for the 2-bit logic gates, and ' $0,0,1$ ', ' $0,1,0$ ' or ' $1,0,0$ ' inputs for the 3 -bit logic gates. When the gate input is set at $I_{2}(=10 \mathrm{~V})$, it represents ' 1,1 ' inputs for the 2-bit logic gates and ' $0,1,1$ ', ' 1 , 0,1 ' or ' $1,1,0$ ' inputs for the 3-bit logic gates. When set at $I_{3}(=15 \mathrm{~V})$, represents ' $1,1,1$ ' inputs for the 3bit logic gates. When gate bias input is OFF, it represents ' 0 ', ' 0,0 ' or ' 0,0 , 0 ' inputs for 1-, 2- or 3-bit logic gates, respectively. The gate input used here consisting of different voltage levels and is different than the conventional transistor based logic gates, which use $\mathrm{N}$-number of independent gate inputs with identical input voltage levels for N-bit logic gates. In this work, the different assigned voltage levels represent the sum of the voltage bias of the $\mathrm{N}$ number of independent voltage gate inputs. Such independent gate inputs can be achieved with $\mathrm{N}$-number of asynchronous square wave generators [12]. Fig. 5(b) shows the frequency response curves of the coupled system for the gate input levels $I_{1}, I_{2}$, and $I_{3}$ are ON. When none of the gate inputs $\left(I_{1}, I_{2}, I_{3}\right)$ are $\mathrm{ON}$, the frequency response curve of the device is shown by the black curve (Fig. 5(b)). The frequency response is due to the DC bias from $V_{B}(55 \mathrm{~V})$, where beams B1 and B2 act as 
parallel plate capacitors and are electrostatically coupled. B1 is positively biased with B2 as the ground (considering the $\mathrm{AC}$ voltage to be negligible in comparison to the $\mathrm{DC}$ voltage). The mode 1 resonance frequency peak here can be used for realising NOT, 2, and 3-bit NOR logic gates, explained in detail later. Mode 2 is not used for realising logic gates due to insufficient DC bias induced shift in resonance frequency curve/peak (Fig. 4), which is essential to achieve switching between the ON and OFF output states (Fig. 1(c)). Next, when $I_{l}$ is ON, the output resonance curve is shown by the red curve (Fig. 5(b)). In this case, the $\mathrm{B} 1$ is biased at $55 \mathrm{~V}$ and $\mathrm{B} 2$ is biased at $5 \mathrm{~V}$. With both beams biased, the overall bias of the system is reduced to $50 \mathrm{~V}$, which causes a change in the stiffness of the coupled beams and shifts the resonance curve to higher frequency. Due to the electrostatic coupling, sufficient shift is seen in the resonance curve of mode 1, whereas the mode 2 curves almost overlap (black versus the red curve). Here, mode 1 is used for realising 2-bit XOR gate. Similarly, when $I_{2}$ or $I_{3}$ is ON, the overall DC bias of the system is reduced to 45 or $40 \mathrm{~V}$, shifting the frequency response to blue and red curves, respectively. The logic gates are realised by driving the device at any of the single desired frequency, where the ON and OFF states are induced by the gate input voltages. Schematic of an XOR logic gate with the required drive frequency, gate inputs and output is shown in Fig. S3 (see supplementary information).

Fig. 6 shows mode 1 time response of the coupled systems as dynamically reconfigurable multifunctional mechanical logic gates. The device is dynamically reconfigured from one logic gate function to another, by simply programing the drive frequency. A 1 - bit NOT gate is realised when the device drive frequency $V_{A C}$ is set at $275.34 \mathrm{kHz}$. The device output is ' 1 ' due to $V_{B}$ even though there is no bias from the gate input. When the gate input is ' 1 ', mode 1 resonance frequency increases to $287.3 \mathrm{kHz}$, changing the device to a non-resonating state at $275.34 \mathrm{kHz}$, functioning as a NOT gate (Fig. 6(a)). At the same frequency, when the inputs are ' 0,0 ', the output is ' 1 ', due to the $V_{B}$. When the inputs are ' 0,1 ', ' 1,0 ', or ' 1,1 ' the output is switched to ' 0 ' providing the output states of a 2-bit NOR logic gate (Fig. 6(b)). Similarly, a 3-bit NOR gate is realised, where, except for ' $0,0,0$ ' gate inputs, the output of the device is always ' 0 ' at the $275.34 \mathrm{kHz}$ (Fig. 6(c)). The system is dynamically changed to a 2-bit XOR gate by 

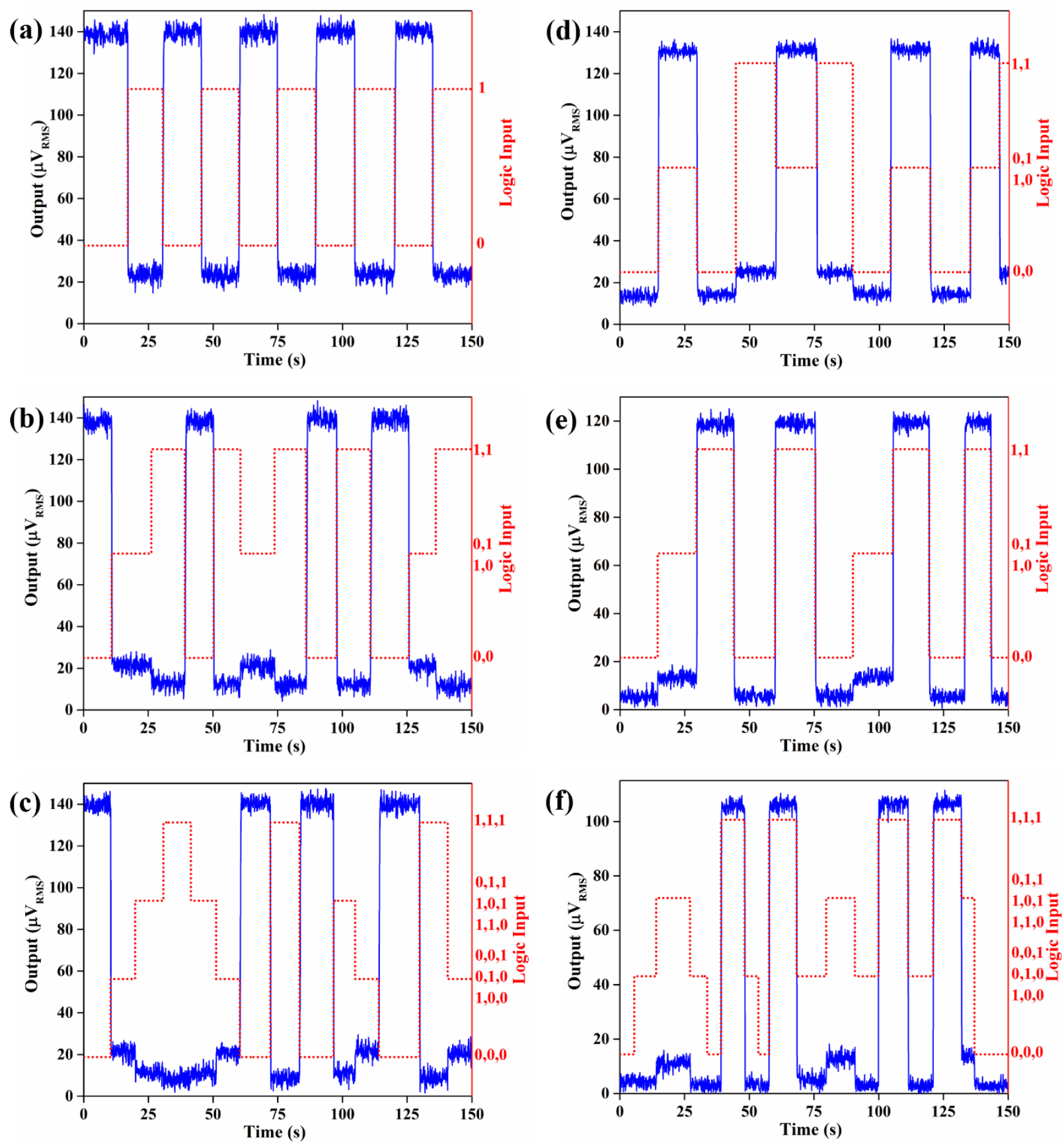

Fig. 6 Time responses of the coupled resonator as a dynamically reconfigurable reduced voltage mechanical logic gate. Gate inputs are represented by red, and the outputs by blue. (a)-(c) Time response of the resonator operating at $275.34 \mathrm{kHz}$ when configured as a NOT, 2-bit NOR and 3-bit NOR logic gates, respectively. (d) Time response of the resonator operating at $287.3 \mathrm{kHz}$ when configured as 2-bit XOR gate. (e), (f) Time response of the resonator operating at $297.1 \mathrm{kHz}$ and $302.72 \mathrm{kHz}$, when configured as 2-bit and 3-bit AND gates, respectively. 
programming $V_{A C}$ to be $287.3 \mathrm{kHz}$. When the gate inputs are ' 0,0 ', or ' 1,1 ', the output state at $287.3 \mathrm{kHz}$ is ' 0 '. When the gate inputs are ' 0,1 ' or ' 1,0 ', the output is switched to ' 1 ', functioning as a 2 -bit XOR gate (Fig. 6(d)). At $V_{A C}=297.1 \mathrm{kHz}$, when the inputs are ' 0,0 ', ' 0,1 ' or ' 1,0 ', the output is ' 0 '. When the gate inputs are ' 1,1 ', the output switches to ' 1 ', functioning as a 2-bit AND gate (Fig. 6(e)). Similarly, a 3bit AND gate is realised at $302.72 \mathrm{kHz}$, where the output state is always ' 0 ' except when the inputs are ' 1 , 1, 1 ' (Fig. 6(f)). Table 1 summarises the truth tables and the operating parameters for realising the different logic gates.

Considering the NOT logic gate, when a $5 \mathrm{~V}$ ('1') gate input is provided, the resonance frequency of mode 1 is shifted by about $11.96 \mathrm{kHz}$ (sufficient to shift the resonant bandwidth and switch between the output states). To achieve a similar shift in resonance frequency of the uncoupled longer beam B2, it would require a DC bias greater than $25 \mathrm{~V}$ as the gate input (for similar drive circuit as shown in Fig. 5(a)). This corroborates that the electrostatic coupling technique reduces the DC bias gate input ' 1 ' by $80 \%$.

\subsection{Dual-band electro-mechanical logic gates}

In addition to the first resonant mode, the second resonant mode can also be used for realizing the logic gates. There are two aspects that define the performance of the first two resonant modes of the device. The first is the difference in the stiffness of the beams arising due to the difference in their lengths. This influences the resonance frequencies and their respective amplitudes at the two modes. The second is the difference in the gaps of the resonating beams with respect to the sense electrode F1. This influences the gain/motional currents of the two modes, where for lower gap the motional current is higher. In other words, higher vibrational amplitudes do not necessarily yield the same $S_{21}$ gain/motional current, since it also depends on the beam's distance from the sense electrode. The combination of these two aspects influences the dynamic electrical response of the device. The first and second resonance frequencies of the coupled system are defined by the resonance frequencies of the uncoupled longer and shorter beams, respectively [21]. Therefore in mode 1, the longer beam has higher amplitude (compared to the amplitude of the shorter beam in mode 1), and in mode 2, the shorter beam has higher amplitude (compared to the amplitude of the 
Table 1. Operating parameters and truth table of the reduced voltage electro-mechanical logic gates.

\begin{tabular}{|c|c|c|c|}
\hline $\begin{array}{l}\text { Operating frequency } \\
\qquad(\mathrm{kHz})\end{array}$ & $\begin{array}{c}\text { Gate input } \\
(\text { Boolean) } \\
0^{\prime}=0 \mathrm{~V} ' 1 '=5 \mathrm{~V}\end{array}$ & $\begin{array}{l}\text { Gate output } \\
\text { (Boolean) }\end{array}$ & $\begin{array}{l}\text { Type of } \\
\text { Logic gate }\end{array}$ \\
\hline \multirow{2}{*}{$275.34($ mode 1$)$} & 0 & 1 & \multirow{2}{*}{ NOT } \\
\hline & 1 & 0 & \\
\hline \multirow{4}{*}{$275.34($ mode 1$)$} & 0,0 & 1 & \multirow{4}{*}{ 2-bit NOR } \\
\hline & 0,1 & 0 & \\
\hline & 1,0 & 0 & \\
\hline & 1,1 & 0 & \\
\hline \multirow{8}{*}{$275.34($ mode 1$)$} & $0,0,0$ & 1 & \multirow{8}{*}{ 3-bit NOR } \\
\hline & $0,0,1$ & 0 & \\
\hline & $0,1,0$ & 0 & \\
\hline & $1,0,0$ & 0 & \\
\hline & $0,1,1$ & 0 & \\
\hline & $1,0,1$ & 0 & \\
\hline & $1,1,0$ & 0 & \\
\hline & $1,1,1$ & 0 & \\
\hline \multirow{4}{*}{$287.3($ mode 1$)$} & 0,0 & 0 & \multirow{4}{*}{ 2-bit XOR } \\
\hline & 0,1 & 1 & \\
\hline & 1,0 & 1 & \\
\hline & 1,1 & 0 & \\
\hline \multirow{4}{*}{$297.1($ mode 1$)$} & 0,0 & 0 & \multirow{4}{*}{ 2-bit AND } \\
\hline & 0,1 & 0 & \\
\hline & 1,0 & 0 & \\
\hline & 1,1 & 1 & \\
\hline \multirow{8}{*}{$302.72($ mode 1$)$} & $0,0,0$ & 0 & \multirow{8}{*}{ 3-bit AND } \\
\hline & $0,0,1$ & 0 & \\
\hline & $0,1,0$ & 0 & \\
\hline & $1,0,0$ & 0 & \\
\hline & $0,1,1$, & 0 & \\
\hline & $1,0,1$ & 0 & \\
\hline & $1,1,0$ & 0 & \\
\hline & $1,1,1$ & 1 & \\
\hline
\end{tabular}

longer beam in mode 2). Further, the coupled system in the first mode always vibrates with higher amplitudes (combined amplitudes of the two beams) compared to the second resonant mode at similar voltages, due to the out-of-phase vibrations leading to higher electrostatic force compared to the in-phase vibrations. As the gate voltage is increased, the electrostatic coupling increases, which softens the beams and thereby reducing their resonance frequencies and increasing the amplitude of vibration [25]. As seen 

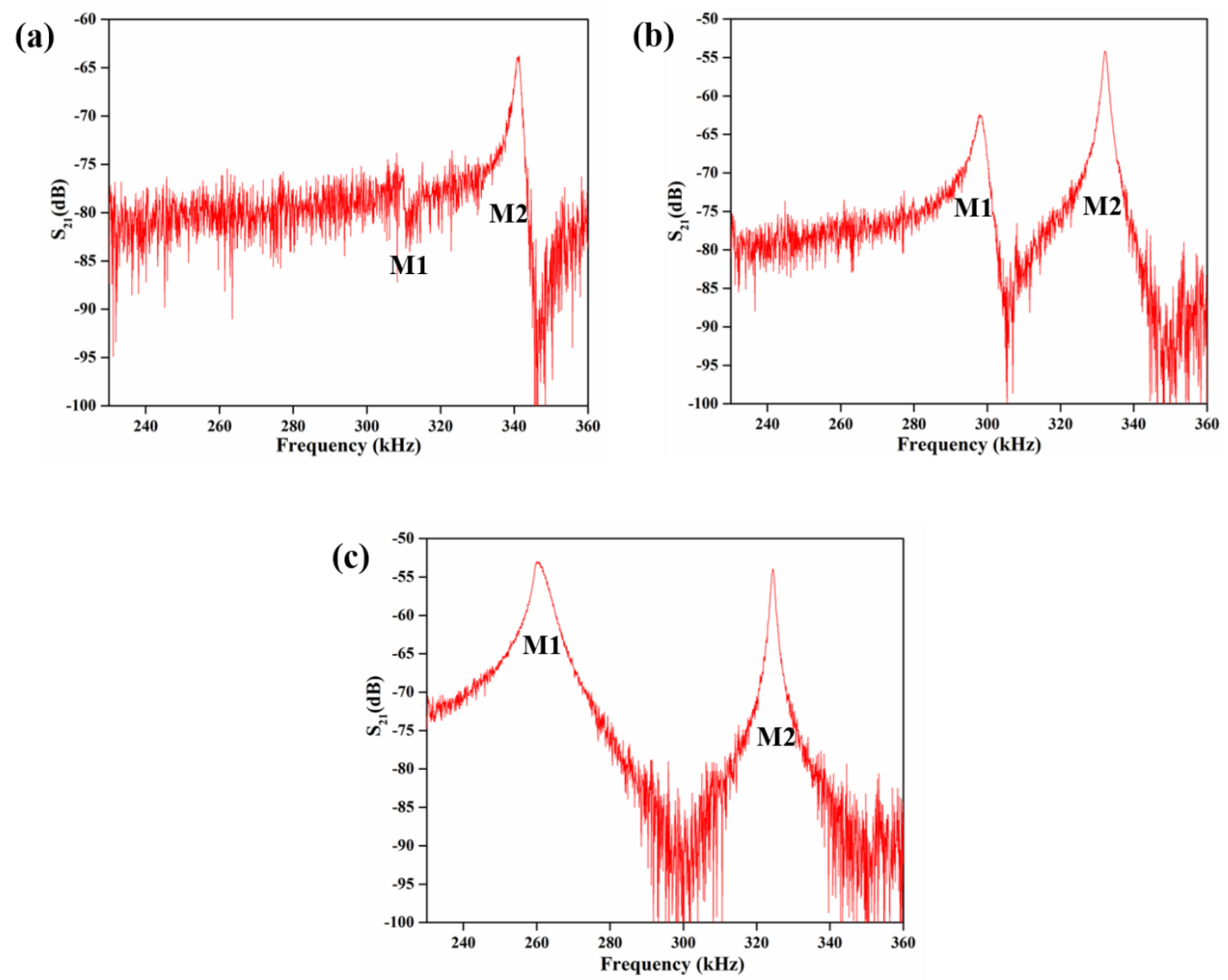

Fig. 7 Frequency response curves of the coupled system at various gate voltages. (a)-(c) Change in the $S_{21}$ response of the coupled cantilevers having two resonant modes (M1 and M2), at 20, 40 and $60 \mathrm{~V}$ gate DC bias, respectively.

in Fig. 7(a), at low gate voltages (below $20 \mathrm{~V})$, the motional current $\left(\mathrm{S}_{21}\right.$ gain) of mode 1 is negligible and buried in the background electrical noise. This is because in mode 1, the shorter beam has low amplitude and its motional current/gain is not sufficiently above the noise level to be detected even though it is at a shorter distance $(2 \mu \mathrm{m})$ from the sense electrode F1. The high amplitude of the longer beam is also not detected due to the higher gap $(7 \mu \mathrm{m})$ from the sense electrode F1. This is in contrary to mode 2 , where the shorter beam has higher amplitude and is closer to the sense electrode. Therefore its motional current is detected at the sense electrode F1. With the increase in the DC gate bias, the motional current for the two 

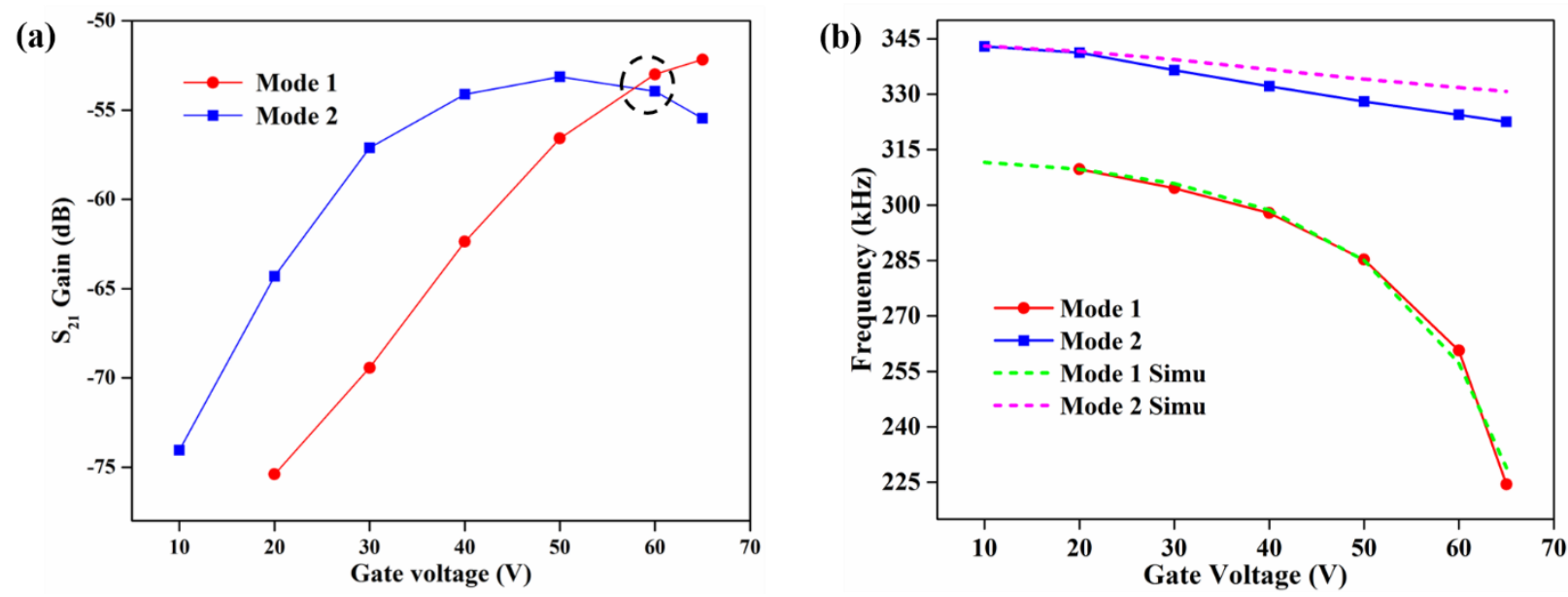

Fig. 8 Comparison of the $S_{21}$ gain and frequency shift of the first two resonant modes (M1 and M2) with the change in gate bias voltage. (a) The change in the $S_{21}$ gain with change in gate bias voltage at the first two resonant modes. (b) The shift in resonance frequency of the first two resonant modes with change in gate bias voltage. The shift in frequency is validated using reduced order model simulations (dashed lines), based on modeling the two beams using an Euler-Bernoulli beam theory [21].

modes increases (Figs. 7(b) and (c)), due to the increased electrostatic loading/coupling and the combined vibrational amplitudes.

The change in $S_{21}$ gain of each resonant mode against the change in the gate bias voltage is shown in Fig. 8 (a). The gain of mode 2 changes nonlinearly from -74 to $-53 \mathrm{~dB}$ when the gate voltage is changed from 10 to $50 \mathrm{~V}$. Above $50 \mathrm{~V}$, the gain of mode 2 starts to decrease, reaching $-55.5 \mathrm{~dB}$ at $65 \mathrm{~V}$. At around $60 \mathrm{~V}$ gate bias, the gain of both modes 1 and 2 overlap at about $-54 \mathrm{~dB}$ (Fig. 8(a), highlighted with black dashed circle). Therefore, both resonant modes can be used for realizing the device as dual-band logic electromechanical gates due to the presence of maximum/equal $S_{21}$ gain at the two resonance frequencies. Identical output states of the two modes of vibration implement logic gates with consistent output levels. The DC bias range was limited to $65 \mathrm{~V}$ as it was close to the dynamic pull-in of the resonator. Fig. 8(b) shows the change in the resonance frequency of the coupled resonator against the change in the gate bias for the two resonant modes. We use this gate induced shift in resonance frequency for switching the device between ON and OFF Boolean states for realizing the dual-band electromechanical logic gates. With the increase in bias voltage, mode 2 shows near-linear decrease in resonance frequency (similar to the 
uncoupled beam, Fig. 4), whereas mode 1 shows nonlinear decrease in resonance frequency. The shift in frequency is due to the electrostatic induced softening of beams B1 and B2 [25]. However, the shift in mode 1 is higher compared to mode 2 due to the difference in the stiffness of the beams as well as the vibrational motion in each mode as explained before. The shift in frequency against the change in gate bias is also verified using a reduced order model based on the Euler-Bernoulli beam theory of a coupled system (dashed lines in Fig. 8 (b)) [21].

Fig. 9 (a) shows the drive circuit for operating the device as a dual-band mechanical logic element. DC bias gate with two voltage input levels $\left(I_{1}, I_{2}\right)$ at 30 and $60 \mathrm{~V}$ are used for defining the gate inputs. The circuit is configured such that when input is set at $I_{1}$, it represents ' 0,1 ' or ' 1,0 ' inputs for the 2-bit logic gates. When set at $I_{2}$, it represents ' 1,1 ' inputs for the 2 - bit logic gates. When the gate input is OFF, it represents ' 0,0 ' inputs for the 2 -bit logic gates. The frequency response of the device for different gate inputs is shown in Fig. 9 (b). The response of the device at 260.4 (mode 1) and 324.4 (mode 2) kHz is used for realizing the two AND gates, and at 304.5 (mode 1) and 336.3 (mode 2) kHz for two XOR logic gates. When the input is ' 0,0 , the beams do not resonate in either of the modes due to the absence of electrostatic loading, resulting in ' 0 ' output at all four frequencies. When the inputs are ' 0,1 ' or ' 1,0 ', the output of the device at 304.5 and $336.3 \mathrm{kHz}$ is ' 1 ', whereas the output at frequencies 260.4 and $324.4 \mathrm{kHz}$ is ' 0 '. When the inputs are ' 1,1 ', the output at frequencies 260.4 and $324.4 \mathrm{kHz}$ is ' 1 ', whereas the output at frequencies 304.5 and $336.3 \mathrm{kHz}$ is ' 0 '. Therefore, at drive frequencies 260.4 and $324.4 \mathrm{kHz}$, the device operates as two AND gate, and at 304.5 and $336.3 \mathrm{kHz}$ as two XOR gates. Figs. 9(c) and (d) show the time sweeps of the device at 260.4 and $336.3 \mathrm{kHz}$, demonstrating the switching operations of AND and XOR gates, respectively. By simply changing the drive frequency, the device is dynamically reconfigurable between any logic gates in real time without the need for any physical change in the drive circuit. Note the output ' 1 ' level at operating frequency $304.5 \mathrm{kHz}$ for ' 0 , 1' or ' 1,0 ' inputs is relatively low. However, with the optimized device dimensions (with reduced difference in the length of the cantilevers) the output signal level may be further enhanced. Table 2 summarises the truth tables and the operating condition for realising the different logic gates. 
(a)

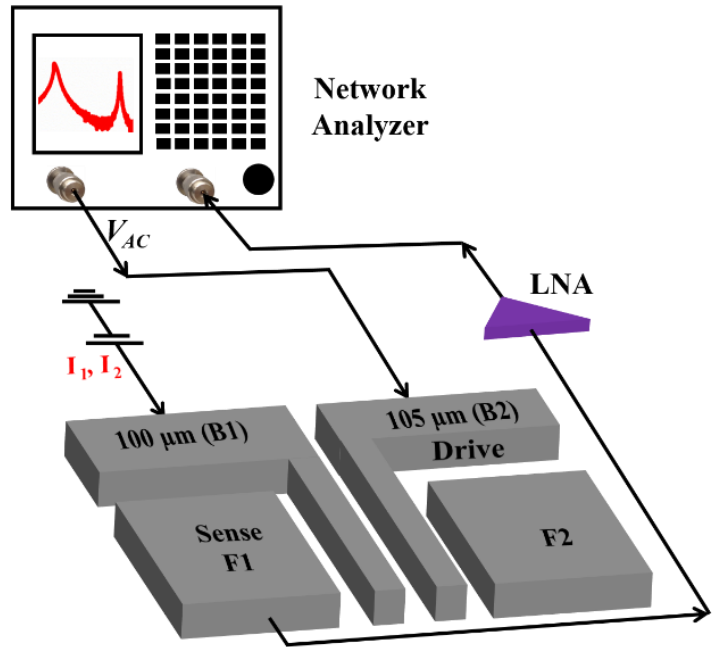

(b)

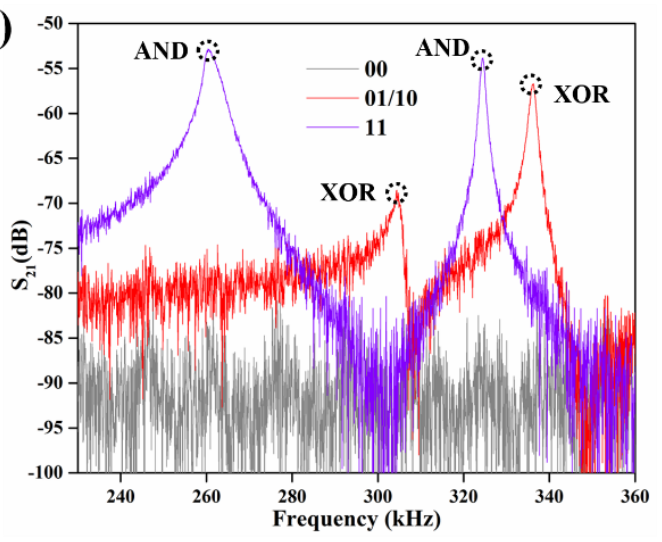

(c)

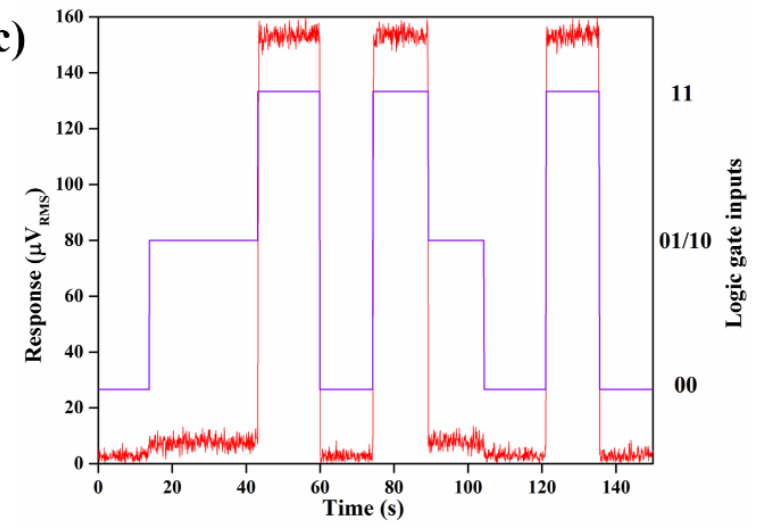

(d)

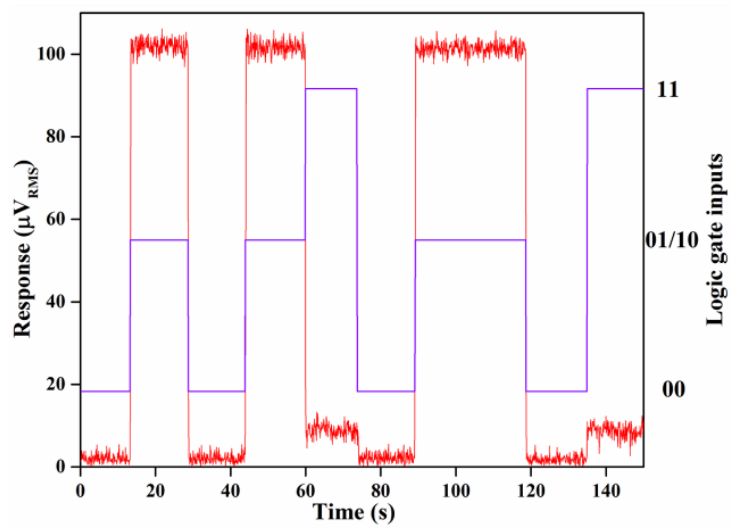

Fig. 9 Dual-band mechanical logic gate. (a) The device drive circuit (b) Frequency response of the device at 0,30 , and $60 \mathrm{~V}$ gate bias. The device bandwidth suitable for operating as two AND and XOR gates are highlighted with dotted circles. (c) Time sweep response of the device as an AND logic gate, operating at $260.4 \mathrm{kHz}$ drive frequency. (d) The time sweep response of the device as an XOR logic gate, operating at $336.3 \mathrm{kHz}$ drive frequency.

\subsection{Device analysis}

The energy cost required by the device to perform one logic operation is given by $E=C V_{B} V_{I}[12]$, where $C$ is the capacitance $\sim 10^{-14} \mathrm{~F}$ (assuming a parallel plate capacitor), $V_{B}$ is the bias voltage (55 V) and $V_{I}$ is the defined gate input voltage. The energy required by the coupled system is $\sim 3 \times 10^{-12} \mathrm{~J}$, whereas that of the uncoupled longer beam is five times higher $\sim 15 \times 10^{-12} \mathrm{~J}(25 \mathrm{~V}$ is defined as the gate input ' 1 '). Therefore, the electrostatic coupling method provides a path for reducing the required actuation voltage and 
Table 2. Operating parameters and truth table of the dual-band electro-mechanical logic gates.

\begin{tabular}{cccc}
\hline \hline $\begin{array}{c}\text { Operating } \\
\text { frequency (kHz) }\end{array}$ & $\begin{array}{c}\text { Gate input (Boolean) } \\
\text { '0'=0V'1'=30 V }\end{array}$ & $\begin{array}{c}\text { Gate output } \\
\text { (Boolean) }\end{array}$ & $\begin{array}{c}\text { Type of } \\
\text { Logic gate }\end{array}$ \\
\hline \hline \multirow{3}{*}{260.4 (mode 1) } & 0,0 & 0 & \\
& 0,1 & 0 & 2-bit AND \\
& 1,0 & 0 & \\
\hline 304.5 (mode 1) & 0,1 & 1 & \\
& 0,1 & 0 & \\
& 1,0 & 1 & 2-bit XOR \\
324.4 (mode 2) & 1,1 & 0 & \\
& 0,0 & 0 & \\
& 0,1 & 0 & 2-bit AND \\
& 1,0 & 0 & \\
336.3 (mode 2) & 1,1 & 1 & \\
& 0,0 & 0 & 2-bit XOR \\
\hline \hline
\end{tabular}

energy of mechanical logic gates. The maximum power of the coupled device is $\sim 8 \mathrm{nW}$ during ON state and $\sim 1 \mathrm{nW}$ during OFF state, defined by $V_{A C} I_{O}$, where $V_{A C}$ is the alternating drive input amplitude (RMS) and $I_{O}$ is the output current before amplification. Although the required power and energy cost per logic operation is relatively higher compared to current transistors [26], they can be significantly reduced by scaling down and optimizing the device geometry. Another important factor affecting the device performance is the switching time per logic operation, which is governed by the required mechanical transition time between the non-resonating and resonating states. This is estimated by $Q / f[11]$, where $Q$ is the quality factor and $f$ is the operating frequency. At $275.34 \mathrm{kHz}$ (mode 1) operating frequency (slowest) and with the quality factor of about 140 , the device requires switching transition time $0.5 \mathrm{~ms}$.

\subsection{Reliability and high temperature performance}

An important factor to consider is the reliability and lifetime of the devices. To demonstrate this, we actuated the device at mode 1 resonance frequency (with $30 \mathrm{~V}$ bias) for 500 hours, using drive circuit as shown in Fig. 3(a). The resonance peaks of the two modes were acquired after every 10 hours of actuation in mode 1. The drift in resonance frequency of mode 1 and 2 are shown in Fig. 10. Mode 1 frequency on 
average is reduced by $60 \mathrm{~Hz}$ per 100 Giga oscillations. This may be due to the reduction in the stiffness of the beams due to the nano-cracks induced during long term actuation. Actuating in mode 1 also affected mode 2 resonance peaks due to the out-of-phase vibration of beams. However, mode 2 showed lower frequency drift of about $15 \mathrm{~Hz}$ on average per 100 Giga oscillations. The full width at half maximum (FWHM) of the resonance curve of mode 1 and 2 are about 3 and $1.2 \mathrm{kHz}$, respectively. Based on the FWHM and drift in resonance frequency, the minimum lifetime of the device at modes 1 and 2 can be estimated to be $5 \times 10^{12}$ and $8 \times 10^{12}$ oscillations, respectively. This demonstrates that the devices have significantly longer lifetime when compared to static actuation lifetime of few switching cycles [21].

One important application where transistors fail to operate is in high temperature environment. To understand the capability of the coupled structure, we studied the temperature variation induced shift in resonance frequency from room temperature up to $150{ }^{\circ} \mathrm{C}$. Figs. 11 (a) and (b) show the temperature induced shift in resonance frequency of mode 1 and mode 2, respectively. Both modes experience an average change of about $13 \mathrm{~Hz}$ per ${ }^{\circ} \mathrm{C}$, which is low compared to clamped-clamped structures. This is due to the absence of temperature induced axial stress, which changes the spring constant of the device. However, the shift in frequency here is due the temperature induced reduction in the Young's modulus of Si [25]. Fig. 11(c) shows the time response of the coupled resonator as an XOR logic at $150{ }^{\circ} \mathrm{C}$. The logic was realized using the drive circuit of Fig. 9 (a) with a drive frequency of $336.4 \mathrm{kHz}$ (mode 2). With a FWHM of $3 \mathrm{kHz}$ at mode 1 , the device is promising to be operational up to $230{ }^{\circ} \mathrm{C}$. However, the experimental measurements were limited to $150{ }^{\circ} \mathrm{C}$ due to the limitation of the experimental setup.

The device demonstrated here operates in the $\mathrm{kHz}$ regime, which is far from the current CMOS devices range. One approach to improve the device performance such as switching speed and integration density is to scale down the device dimensions. This can be achieved by using e-beam lithography [14] instead of optical lithography, which can reduce the dimensions of the device to as low as $50 \mathrm{~nm}$. Such devices can have resonance frequencies up to few tens of MHz. The frequency can be further increased by using clamped-clamped structures instead of cantilevers. However, clamped-clamped structures are more prone to temperature induced shift in resonance frequency, limiting the operating temperature range. Also, 

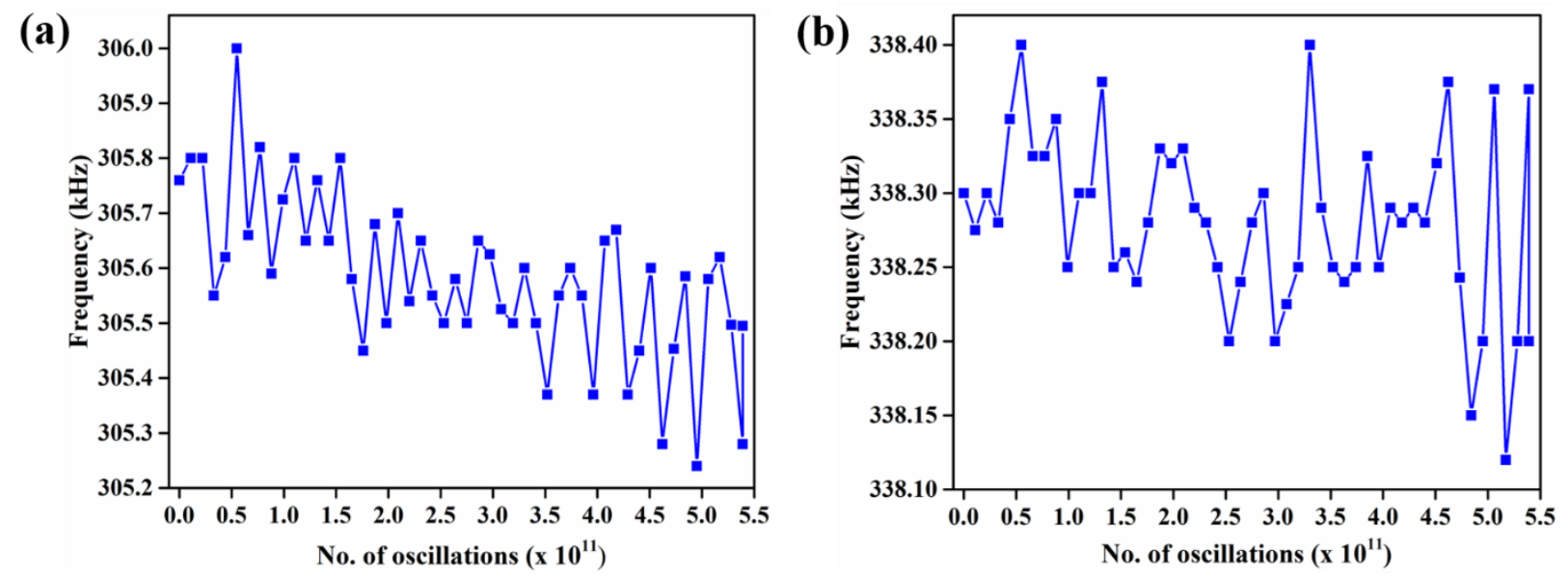

Fig. 10 Reliability study of the proposed device. (a) Resonance frequency drift in mode 1 over 500 hours of operation. (b) Resonance frequency drift in mode 2 over 500 hours of operation in mode 1.

(a)

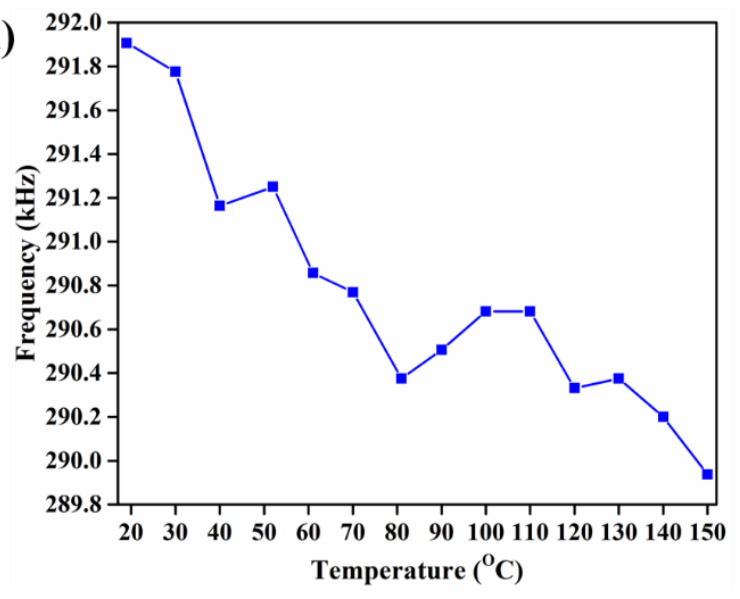

(b)

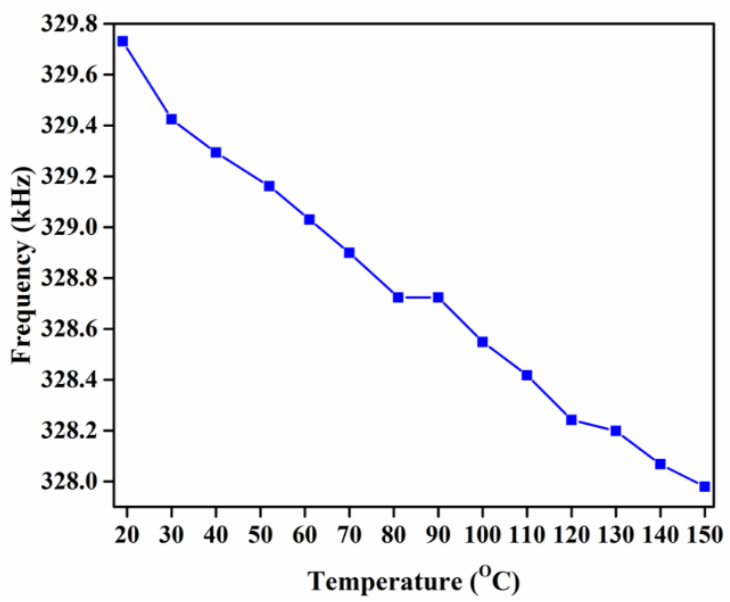

(c)

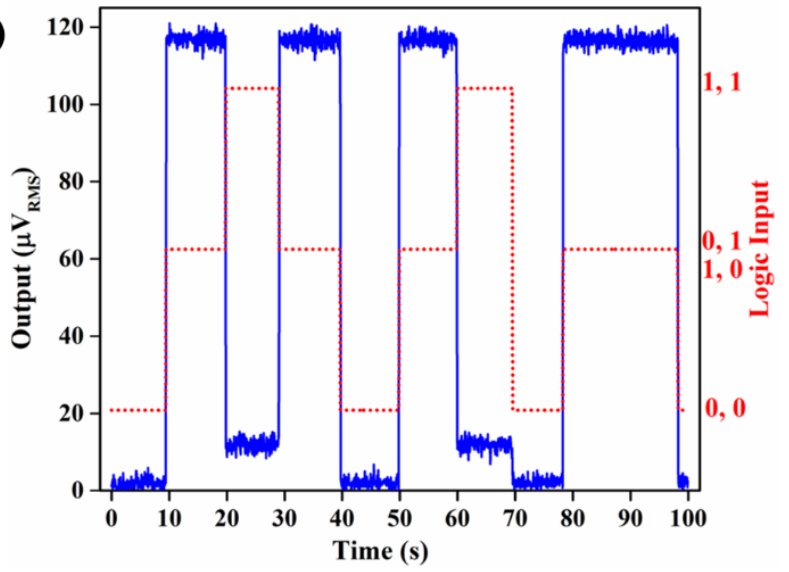

Fig. 11 Change in resonance frequency with temperature. (a) Temperature variation induced shift in mode 1 in resonance frequency. (b) Temperature variation induced shift in mode 2 in resonance frequency. (c) Time response of the coupled resonator as a XOR logic operating at $150{ }^{\circ} \mathrm{C}$. 
increasing the resonance frequency may increase the actuation voltage of the devices leading to higher power consumption. The actuation voltage can be reduced by reducing the actuation gap of these devices in addition to the demonstrated electrostatic coupling approach. Another approach to increase the resonance frequency is to use stiffer materials such as $1 \mathrm{~d}$ carbon nanotubes. They are hollow and highly stiff, providing high frequency and lower actuation voltages with resonance frequency up to few GHz [27]. However, the key challenge is to fabricate devices with carbon nanotubes that are aligned and near identical to each other, such that the desired electrostatic coupling can be achieved. Another effect of scaling down the device is reduction in the overlap area (for electrostatic coupling). This will be lead to reduced signal to noise ratio limiting the detected output signal. Realising such devices could be comparable to current CMOS devices in terms of speed and power.

\section{Conclusions}

We have presented the electrical characterization of two near identical electrostatically coupled microcantilever beam resonators. The device has two resonant modes, where in the first mode the two beams vibrate out-of-phase and in the second mode they vibrate in-phase. Due to electrostatic coupling, mode 1 has 335\% higher shift in DC bias induced shift in resonance frequency compared to the uncoupled system. Utilizing the larger gate bias (at $40 \mathrm{~V}$ and above) induced shift in mode 1 resonance frequency, 2-bit XOR, 2- and 3-bit AND, 2- and 3-bit NOR, and 1-bit NOT mechanical logic gates were demonstrated. By electrostatic coupling, the required gate voltage is reduced by $80 \%$, and thereby reducing the energy cost per logic operation. The device can be dynamically switched between gates function by simply programming the drive frequency and without the need for any change in the drive circuit. Alternatively, using the optimized response of the first two resonant modes of the coupled device, we demonstrated dual 2-bit AND and dual 2-bit XOR logic gates within the same device, providing increased operating frequency bandwidth and functionality. The device has a switching time of about $0.5 \mathrm{~ms}$ per logic operation, operating at 5 Torr pressure. The device was demonstrated operational from room temperature up to $150{ }^{\circ} \mathrm{C}$, making 
it a promising candidate for high temperature logic gates. The device performance can be significantly enhanced by scaling down its dimensions. Such a device provides a promising path towards scalable mechanical computational architectures with reduced energy, enhanced computing capabilities and functionalities.

\section{Competing interest}

The authors declare that they have no competing interests.

\section{Acknowledgment}

This work has been supported through King Abdullah University of Science and Technology (KAUST) research funds. We like to thank Professor Pedro da Costa for the help with the experimental test setup and Manya Chappanda for her help with coloring the SEM image.

\section{References}

[1] Biswas A, Bayer I S, Biris A S, Wang T, Dervishi E and Faupel F 2012 Advances in top-down and bottom-up surface nanofabrication: Techniques, applications \& future prospects Adv. Colloid Interface Sci. $1702-27$.

[2] Lee T-H, Bhunia S and Mehregany M 2010 Electromechanical Computing at $500^{\circ} \mathrm{C}$ with Silicon Carbide Science 329 1316-1318.

[3] Jaeseok J, Pott V, Kam H, Nathanael R, Alon E and Liu T-J K 2010 Seesaw relay logic and memory circuits J. Microelectromech. Syst. 19 1012-1014.

[4] Sinha N, Jones T S, Guo Z and Piazza G 2012 Body-biased complementary logic implemented using AlN piezoelectric MEMS switches J. Microelectromech. Syst. 21 484-496.

[5] Ilyas S, Arevalo A, Bayes E, Foulds I G and Younis M I 2015 Torsion based universal MEMS logic device Sens. Actuat. A-Phys. 236 150-158. 
[6] Chowdhury F K, Chappanda K N, Saab D and Tabib-Azar M 2011 Novel single-device XOR and AND gates for high speed, very low power LSI mechanical processors Int. Conf. on Solid-State Sensors, Actuators and Microsystems pp 1100-1103.

[7] Tsai C-Y, Kuo W-T, Lin C-B and Chen T-L 2008 Design and fabrication of MEMS logic gates $J$. Micromech. Microeng. 18045001.

[8] Tsai C-Y and Chen T-L 2010 Design, fabrication and calibration of a novel MEMS logic gate $J$. Micromech. Microeng. 20095021.

[9] Yao A and Hikihara T Logic-memory device of a mechanical resonator 2014 Appl. Phys. Lett. 105, p 123104.

[10] Ilyas S, Jaber N and Younis M I 2017 MEMS logic using mixed-frequency excitation $J$. Microelectromech. Syst. 26 1140-1146.

[11] Wenzler J-S, Dunn T, Toffoli T and Mohanty P 2014 A Nanomechanical Fredkin Gate Nano Lett. 14 89-93.

[12] Guerra D N, Bulsara A R, Ditto W L, Sinha S, Murali K and Mohanty P 2010 A Noise-Assisted Reprogrammable Nanomechanical Logic Gate Nano Lett. 10 1168-1171.

[13] Mahboob I, Flurin E, Nishiguchi K, Fujiwara A and Yamaguchi H 2011 Interconnect-free parallel logic circuits in a single mechanical resonator Nat. Commun. 2198.

[14] Chappanda K N, Ilyas S, Kazmi S N R, Holguin-Lerma J, Batra N M, Costa P M F J and Younis M I 2017 A single nano cantilever as a reprogrammable universal logic gate J. Micromech. Microeng. 27 045007.

[15] Kazmi S N R, Hafiz M A A, Chappanda K N, Ilyas S, Holguin-Lerma J, Costa P M F J and Younis M I 2017 Tunable nanoelectromechanical resonator for logic computations Nanoscale 9 3449-3457.

[16] Hatanaka D, Mahboob I, Okamoto H, Onomitsu K and Yamaguchi H 2012 An electromechanical membrane resonator Appl. Phys. Lett. 101063102.

[17] Masmanidis S C, Karabalin R B, Vlaminck I D, Borghs G, Freeman M R and Roukes M L 2007 Multifunctional nanomechanical systems via tunably coupled piezoelectric actuation Science 317 780-783. 
[18] Hafiz M A A, Kosuru L and Younis M I 2016 Towards electromechanical computation: An alternative approach to realize complex logic circuits J. Appl. Phys. 120074501.

[19] Hafiz M A A, Kosuru L, Ramini A, Chappanda K N and Younis M I 2016 In-Plane MEMS Shallow Arch Beam for Mechanical Memory Micromachines 7191.

[20] Chappanda K N and Tabib-Azar M 2011 Conducting AFM studies of metal surface contact resistance for NEMS switches Proc. IEEE Sensors pp 1371-1373.

[21] Ilyas S, Chappanda K N, Hafiz M A A, Ramini A and Younis M I 2016 An experimental and theoretical investigation of electrostatically coupled cantilever microbeams Sens. Actuat. A-Phys. 247 368-378.

[22] Ilyas S, Jaber N and Younis M I 2016 Static and Dynamic Amplification Using Strong Mechanical Coupling J. Microelectromech. Syst. 25 916-921.

[23] Siavash P P and Ayazi F 2005 Electrically coupled MEMS bandpass filters: part II. Without coupling element Sens. Actuat. A-Phys. 122 317-325.

[24] Ilyas S, Chappanda K N and Younis M I 2017 Exploiting nonlinearities of micro-machined resonators for filtering applications Appl. Phys. Lett. 110253508.

[25] Younis M I 2011 MEMS Linear and Nonlinear Statics and Dynamics (New York: Springer)

[26] Lee S and Nathan A 2016 Subthreshold Schottky-barrier thin-film transistors with ultralow power and high intrinsic gain Science 354 302-304.

[27] Laird E A 2012 A High Quality Factor Carbon Nanotube Mechanical Resonator at 39 GHz Nano Lett. 12, 193-197. 


\title{
Supplementary information
}

\section{Micro-mechanical Resonators for Dynamically Reconfigurable Reduced Voltage Logic Gates}

\author{
K. N. Chappanda ${ }^{1}$, S. Ilyas ${ }^{1}$, and M. I. Younis ${ }^{1 *}$ \\ ${ }^{1}$ Nano/Micro Mechanics and Motion Laboratory, Physical Science and Engineering Division, King \\ Abdullah University of Science and Technology, Thuwal, 23955-6900, Kingdom of Saudi Arabia. \\ "Corresponding author e-mail: mohammad.younis@kaust.edu.sa
}

Device fabrication: 4-inch silicon-on-insulator (SOI) wafer from Semiconductor Wafer Inc was degreased by ultra-sonicating in acetone, ethanol and water. The wafer was then blow-dried in nitrogen gas. First photolithography step was used for defining the metallization layer. Metallization was used to reduce the electrical contact resistance during probing/characterizing the device. AZ1512 positive photoresist was used spun coated at 3000 RPM to form $1.5 \mu \mathrm{m}$ thick film. The resist was exposed and developed, defining the metallization layer. $50 \mathrm{~nm}$ of $\mathrm{Cr}$ was first DC sputter deposited followed by $250 \mathrm{~nm}$ of $\mathrm{Au}$ at $400 \mathrm{~W}$ power. $\mathrm{Cr}$ was used as adhesion layer for $\mathrm{Au}$. Lift-off process was used to pattern the $\mathrm{Cr} / \mathrm{Au}$ film. A second photolithography was used to define the beams and electrodes. ECI 3027 positive photoresist was spun coated at $1500 \mathrm{RPM}$ to form $4 \mu \mathrm{m}$ thick film. The wafer was then subjected to Bosch deep reactive ion etching (DRIE) process to etch the device Si layer. The process consisted of 80 alternating cycles of $7 \mathrm{~s}$ long side wall passivation using $\mathrm{C}_{4} \mathrm{~F}_{8}$ and $6 \mathrm{~s}$ plasma $\mathrm{Si}$ etch using $\mathrm{SF}_{6}$ at 20 mTorr pressure. The wafer was maintained at $-20{ }^{\circ} \mathrm{C}$ during the entire DRIE process. The number of cycles were optimized such that the Si layer is etched exposing the buried oxide layer. The beams were released by etching the buried oxide layer in $\mathrm{HF}$ vapor at $40{ }^{\circ} \mathrm{C}$ for 45 mins. 


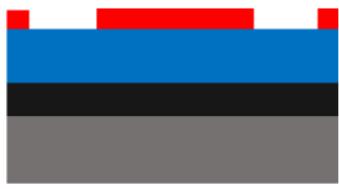

Define the metallization layer using optical lithography

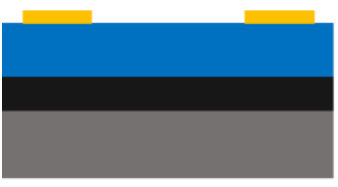

Sputter deposit $50 / 250 \mathrm{~nm}$ of $\mathrm{Cr} / \mathrm{Au}$ layer followed by lift-off

Define beams/electrodes

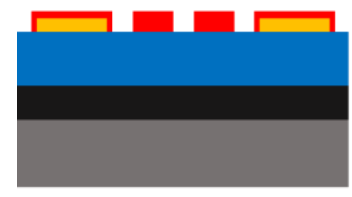
using optical lithography
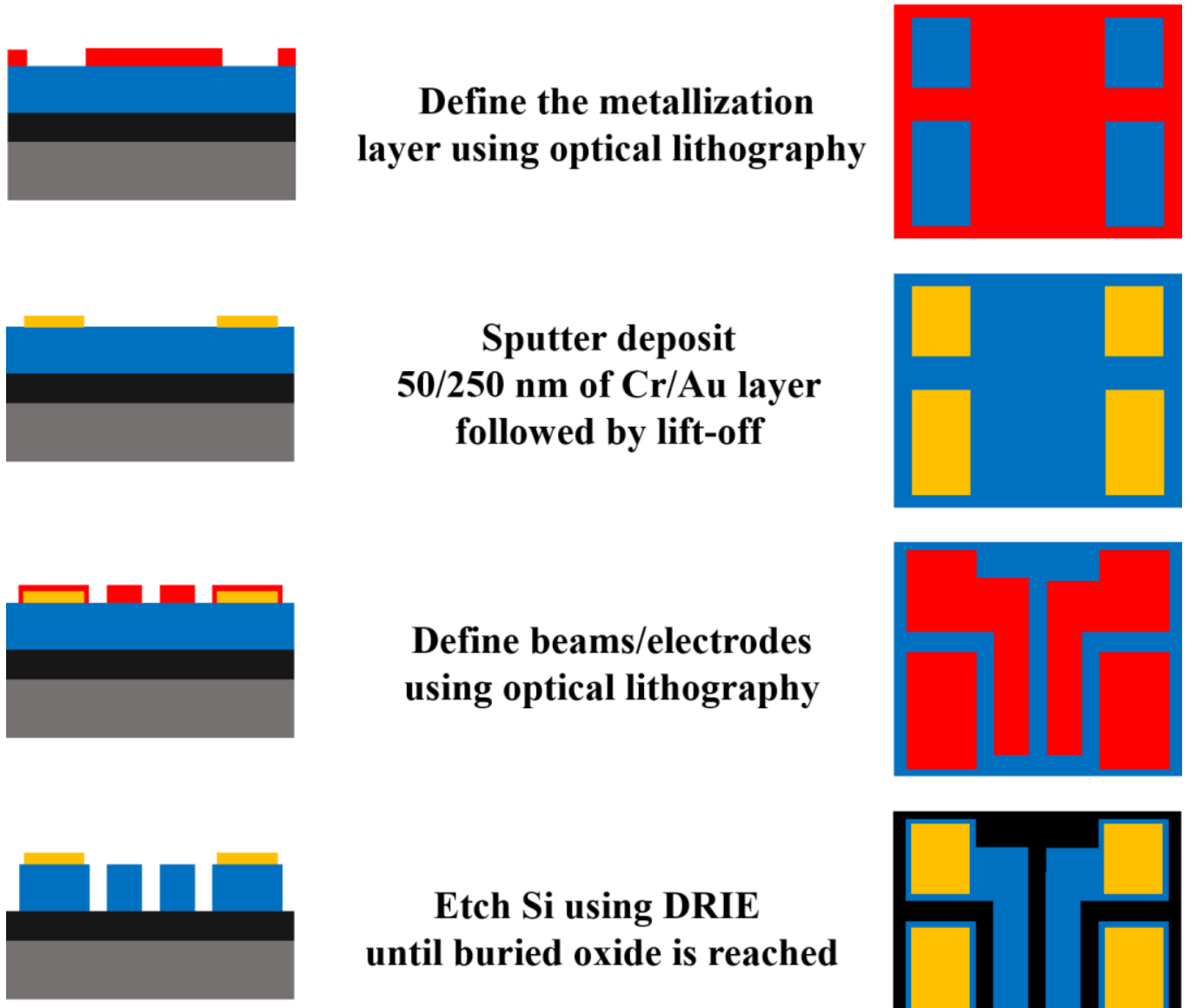

Etch Si using DRIE until buried oxide is reached
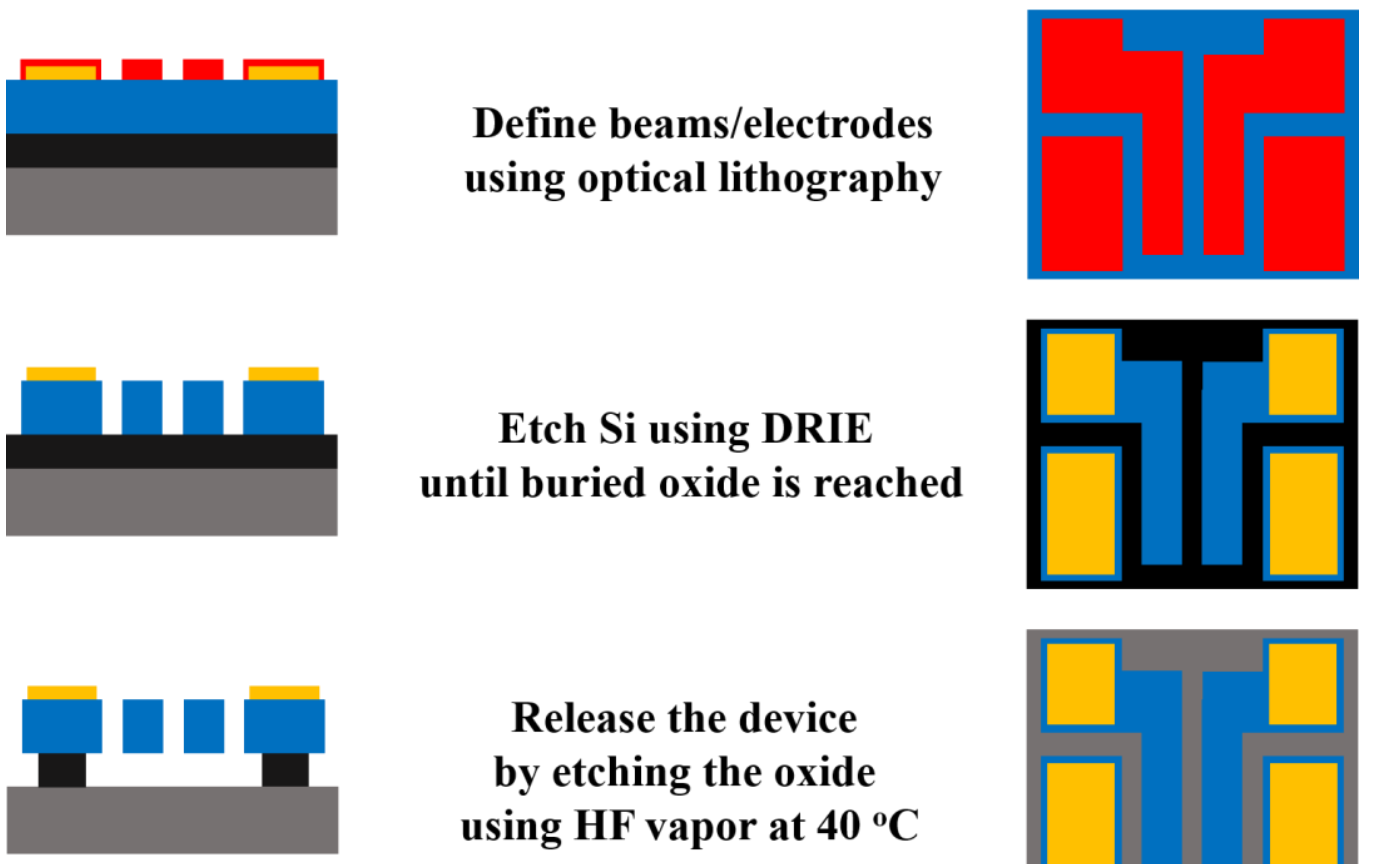

Release the device by etching the oxide using $\mathrm{HF}$ vapor at $40{ }^{\circ} \mathrm{C}$
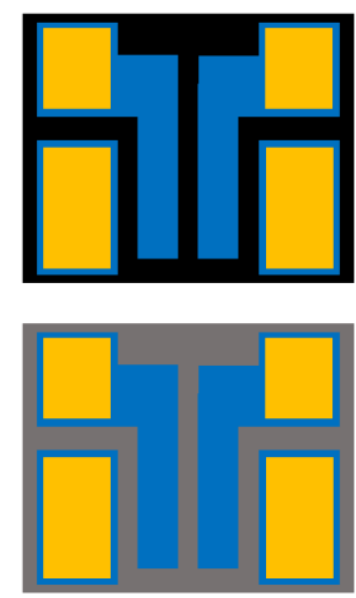

- Si substrate

Buried oxide layer

- Si device layer

Photoresist

\section{Metallization (Cr/Au)}

Figure S1: Schematic of the steps used for fabricating coupled Si micro-cantilever beams. 
$\mathbf{a}$

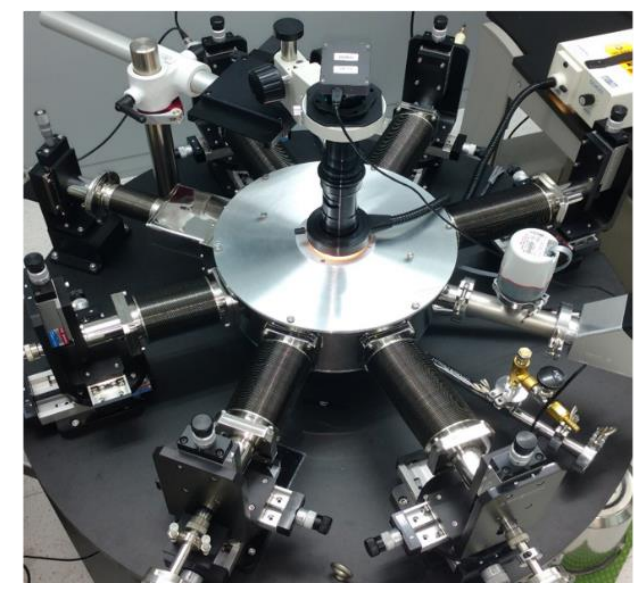

b

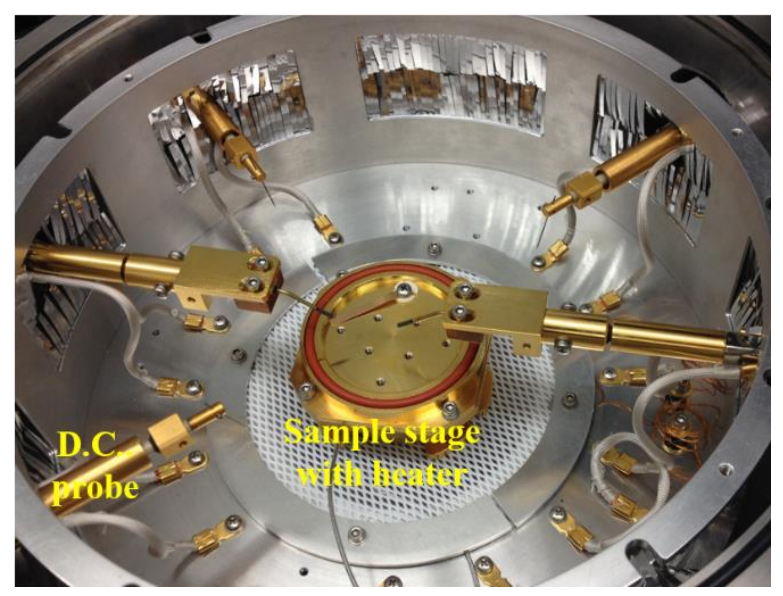

Figure S2: Digital image of the ST-500 temperature controlled vacuum probe station from Janis Research used for demonstrating the logic gates at room and elevated temperatures. (a) Outside view of the probe station. (b) Inside view of the probe station. Three DC probes were used for characterizing the devices.

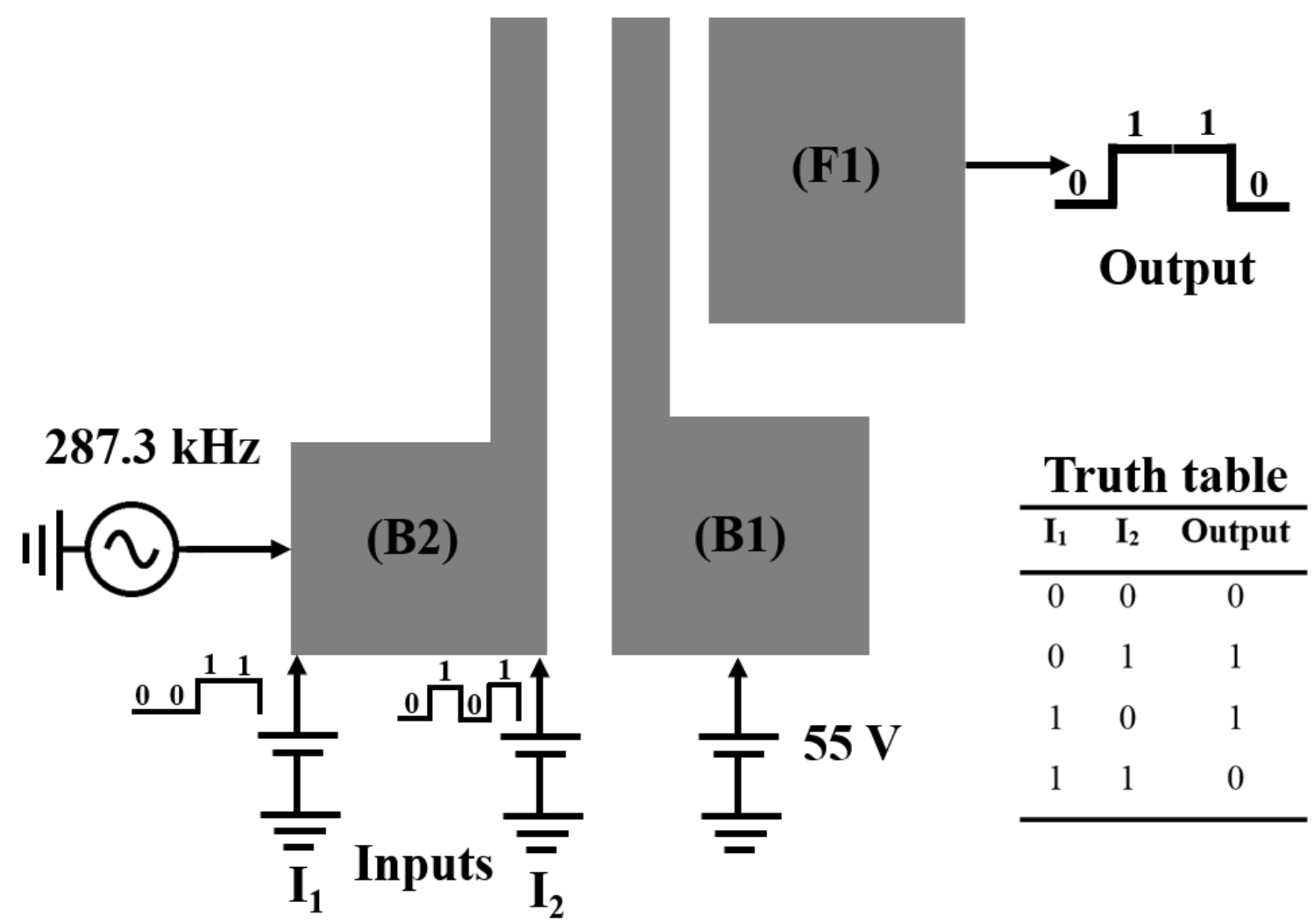

Figure S3: Schematic of an XOR logic gate based on dynamic actuation. The ON and OFF output state of logic device is defined by the resonating and non-resonating state of the coupled beams at drive frequency. 
The drive frequency is set at $287.3 \mathrm{kHz}$. The gate inputs $\mathrm{I}_{1}$ and $\mathrm{I}_{2}$ are interfaced to the longer cantilever $\mathrm{B} 2$. The shorter cantilever B1 is biased with $55 \mathrm{~V}$. When either of the inputs are $\mathrm{ON}$, the device resonance is at drive frequency yielding ON output state at the sense electrode F1. When both or none of the inputs are $\mathrm{ON}$, the device resonance is away from the drive frequency, resulting in OFF output state at F1. The function of the logic gate can be changed by simply changing the drive frequency without the need for any change in the circuit. 